\title{
High energy resolution fluorescence XAFS for the speciation of trace elements in geochemical and environmental samples
}

\author{
YOSHIO TAKAHASHI AND AKIKO YAMAGUCHI
}

The University of Tokyo

Presenting Author: ytakaha@eps.s.u-tokyo.ac.jp

Speciation of trace elements in environment is essential for better understanding of their behaviors in environment. For this purpose, X-ray absorption fine structure spectroscopy (XAFS) in fluorescence mode is one of most effective methods, but interference on the signal of each trace element by those from background elements is a severe problem to selectively obtain the signal from trace elements. To overcome the difficulties, we have employed two methods, which use (i) high energy resolution energy dispersive detector and (ii) wavelength dispersive detection system. In this presentation, various applications of the two methods were shown for chemical state analysis of trace elements in geochemical samples. For example, oxidation state of $\mathrm{Eu}$ in feldspar, oxidation state of $\mathrm{Ce}$ in weathered granite, and radiocesium (RCs) species in cesiumbearing silica microparticles (CsMP) using X-ray absorption near-edge structure (XANES) analysis in fluorescence mode. It is also evident that to measure extended X-ray absorption fine structure (EXAFS) spectroscopy is not possible due to the lower signal to noise ratio for many geochemical and environmental samples. On the other hand, XANES, which can be obtained for trace elements with better sensitivity, is not very informative in terms of chemical state analysis of various elements in the samples. However, higher energy resolution detection of fluorescence X-ray than the degree of lifetime broadening enables us to measure XANES itself by better energy resolution, which is known as high energy resolution fluorescence detection (HERFD) XANES. The HERFD-XANES has more information on electronic state of each element given by the method, by which we can extract chemical information to the maximum degree possible. As an example of HERFD-XANES, speciation of cesium and barium adsorbed within the interlayer of phyllosilicates will be presented. 